Jia-Zhen Wang, Wei-Zhao Weng, Ying Ma, Xiu-Ting He and Qing-Guo Meng*

\title{
Crystal structure of $(1 S, 3 \mathrm{a} R, 3 \mathrm{~b} R, 10 \mathrm{a} R, 10 \mathrm{~b} R, 12 \mathrm{a} R)-8$-amino-3a,3b,6,6,10a- pentamethyl-1-((S)-2,6,6-trimethyltetrahydro-2H-pyran-2-yl)-2,3,3a,3b, 4,5,5a,6,10,10a,10b,11,12,12a-tetradecahydro-1H-cyclopenta[7,8] phenanthro[2,3-d]thiazol-12-ol - a panaxadiol dervative, $\mathrm{C}_{31} \mathrm{H}_{50} \mathrm{~N}_{2} \mathrm{O}_{2} \mathrm{~S}$
}

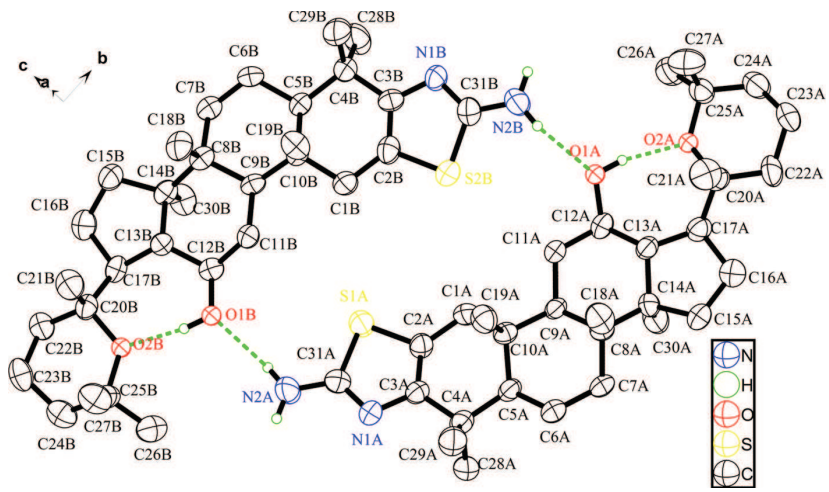

https://doi.org/10.1515/ncrs-2018-0238

Received October 17, 2018; accepted October 26, 2018; available online February 4, 2019

\section{Abstract \\ $\mathrm{C}_{31} \mathrm{H}_{50} \mathrm{~N}_{2} \mathrm{O}_{2} \mathrm{~S}$, orthorhombic, $P 22_{1} 2_{1} 2_{1}$ (no. 19), $a=11.9922(9) \AA$, $b=16.7099(8) \AA, c=29.1262(16) \AA, V=5836.6(6) \AA^{3}, Z=8$, $R_{g t}(F)=0.0654, w R_{\text {ref }}\left(F^{2}\right)=0.1230, T=293(2) \mathrm{K}$.}

\section{CCDC no.: 1858965}

The asymmetric unit, consisting of two independent title molecules, of the crystal structure is shown in the figure. Tables 1 and 2 contain details on crystal structure and measurement conditions and a list of the atoms including atomic coordinates and displacement parameters.

\section{Source of material}

Panaxadiol (PD; systematic name: (3S,5R,8R,9R,10R,12R,13R, $14 R, 17 S)-4,4,8,10,14$-pentamethyl-17- $((R)-2,6,6$-trimethyltetra-

*Corresponding author: Qing-Guo Meng, School of Pharmacy, Collaborative Innovation Center of Advanced Drug Delivery System and Biotech Drugs in Universities of Shandong, Key Laboratory of Molecular Pharmacology and Drug Evaluation (Yantai University), Ministry of Education, Yantai University, Yantai Province 264005, P.R. China, e-mail: qinggmeng@163.com

Jia-Zhen Wang, Wei-Zhao Weng, Ying Ma and Xiu-Ting He: School of Pharmacy, Collaborative Innovation Center of Advanced Drug Delivery System and Biotech Drugs in Universities of Shandong, Key Laboratory of Molecular Pharmacology and Drug Evaluation (Yantai University), Ministry of Education, Yantai University, Yantai Province 264005, P.R. China
Table 1: Data collection and handling.
Crystal:

Size:

Wavelength:

$\mu$ :

Diffractometer, scan mode:

$\theta_{\max }$, completeness:

$N(h k l)_{\text {measured }}, N(h k l)_{\text {unique }}, R_{\text {int }}$ :

Criterion for $I_{\mathrm{obs}}, N(h k)_{\mathrm{gt}}$ :

$N(\text { param })_{\text {refined }}$ :

Programs:

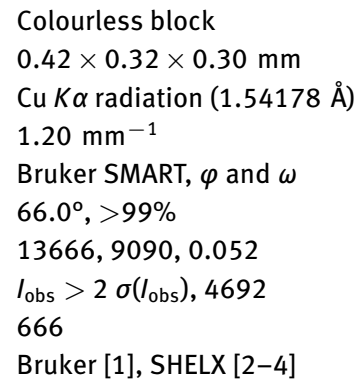

Colourless block

$0.42 \times 0.32 \times 0.30 \mathrm{~mm}$

Cu $K \alpha$ radiation $(1.54178 \AA$ A

$1.20 \mathrm{~mm}^{-1}$

Bruker SMART, $\varphi$ and $\omega$

$66.0^{\circ},>99 \%$

$13666,9090,0.052$

$I_{\text {obs }}>2 \sigma\left(I_{\text {obs }}\right), 4692$

666

Bruker [1], SHELX [2-4]

hydro-2H-pyran-2-yl)hexadecahydro- $1 H$-cyclopenta $[a]$ phenanthrene-3,12-diol) was isolated from the degradation residues of Panax ginseng treated with $10 \% \mathrm{H}_{2} \mathrm{SO}_{4}$, and purified by silica gel column chromatography. After recrystallization in ethyl acetate and $\mathrm{CH}_{2} \mathrm{Cl}_{2}$, the pure white needle - like crystal line product was obtained. PD was oxidized by pyridinium chlorochromate in dry dichloromethane to get the 3-Oxo-PD; then the products occur bromine reaction with pyridinium tribromide in acetic acid and stirred at room temperature to get the 2-bromo-3-oxo-PD. 2-Bromo3-oxo-PD, thiourea was added in ethanol, stirred at $80^{\circ} \mathrm{C}$ for $8 \mathrm{~h}$. The solution was then washed successively with water and saturated with sodium chloride solution, and dried over anhydrous sodium sulfate. After filtration, the ethyl acetate was evaporated in vacuo to yield a white solid, which was purified using silica-gel column chromatography (petroleum ether:ethyl acetate $=3: 1, v / v$ ). Crystals were obtained by slow concentration of an ethyl acetate solution. HRMS calcd. for $\mathrm{C}_{31} \mathrm{H}_{50} \mathrm{~N}_{2} \mathrm{O}_{2} \mathrm{~S}[\mathrm{M}+\mathrm{H}]+515.36$; found 515.33 . ${ }^{1} \mathrm{H}$ NMR $\left(400 \mathrm{MHz}, \mathrm{CDCl}_{3}\right) \delta 6.31(\mathrm{~s}, 1 \mathrm{H}), 3.55(\mathrm{tt}, J=10.3$, $4.9 \mathrm{~Hz}, 1 \mathrm{H}), 2.58(\mathrm{~d}, J=15.4 \mathrm{~Hz}, 1 \mathrm{H}), 2.17(\mathrm{~d}, J=15.2 \mathrm{~Hz}, 1 \mathrm{H})$, 1.98 (s, 2H), 1.77 (dd, $J=22.3,11.4 \mathrm{~Hz}, 2 \mathrm{H}), 1.66(\mathrm{dd}, J=19.9$, $9.2 \mathrm{~Hz}, 1 \mathrm{H}), 1.59$ (d, $J=12.7 \mathrm{~Hz}, 6 \mathrm{H}), 1.51-1.45(\mathrm{~m}, 2 \mathrm{H})$, $1.41-1.30$ (m, 3H), 1.26 (s, 3H), 1.24 (d, $J=2.3 \mathrm{~Hz}, 3 \mathrm{H}), 1.20$ $(\mathrm{s}, 6 \mathrm{H}), 1.19(\mathrm{~s}, 3 \mathrm{H}), 1.11(\mathrm{~d}, J=3.5 \mathrm{~Hz}, 3 \mathrm{H}), 1.02(\mathrm{~d}, J=2.5 \mathrm{~Hz}$, $3 \mathrm{H}), 0.93(\mathrm{~d}, J=2.5 \mathrm{~Hz}, 3 \mathrm{H}), 0.89(\mathrm{~d}, J=2.5 \mathrm{~Hz}, 6 \mathrm{H})$.

\section{Experimental details}

All $\mathrm{H}$ atoms were included in calculated positions and refined as riding atoms, with $\mathrm{O}-\mathrm{H}=0.82 \AA(-\mathrm{OH}), U_{\text {iso }}$ 
Table 2: Fractional atomic coordinates and isotropic or equivalent isotropic displacement parameters $\left(\AA^{2}\right)$.

\begin{tabular}{|c|c|c|}
\hline Atom & $x$ & $y$ \\
\hline $\mathrm{N} 1 \mathrm{~A}$ & $0.5240(5)$ & $0.0172(4)$ \\
\hline $\mathrm{N} 2 \mathrm{~A}$ & $0.5564(5)$ & $-0.0442(3)$ \\
\hline $\mathrm{H} 2 \mathrm{~A} 1$ & 0.564385 & -0.090852 \\
\hline $\mathrm{H} 2 \mathrm{~A} 2$ & 0.562238 & -0.038141 \\
\hline $01 \mathrm{~A}$ & $0.5350(5)$ & $0.5452(3)$ \\
\hline $\mathrm{H} 1 \mathrm{~A}$ & 0.527543 & 0.591195 \\
\hline $02 \mathrm{~A}$ & $0.4914(4)$ & $0.6838(3)$ \\
\hline S1A & $0.51516(19)$ & $0.11615(12)$ \\
\hline $\mathrm{C} 1 \mathrm{~A}$ & $0.4800(6)$ & $0.2399(4)$ \\
\hline H1A1 & 0.520914 & 0.271623 \\
\hline $\mathrm{H} 1 \mathrm{~A} 2$ & 0.401860 & 0.254408 \\
\hline $\mathrm{C} 2 \mathrm{~A}$ & $0.4938(6)$ & $0.1533(4)$ \\
\hline C $3 \mathrm{~A}$ & $0.5022(6)$ & $0.0923(4)$ \\
\hline $\mathrm{C} 4 \mathrm{~A}$ & $0.4923(7)$ & $0.1036(5)$ \\
\hline C $5 \mathrm{~A}$ & $0.4721(6)$ & $0.1942(4)$ \\
\hline $\mathrm{H} 5 \mathrm{~A}$ & 0.391524 & 0.201595 \\
\hline $\mathrm{C} 6 \mathrm{~A}$ & $0.4948(8)$ & $0.2142(4)$ \\
\hline H6A1 & 0.574229 & 0.210444 \\
\hline $\mathrm{H} 6 \mathrm{~A} 2$ & 0.457488 & 0.175231 \\
\hline C7A & $0.4547(7)$ & $0.2975(4)$ \\
\hline H7A1 & 0.374004 & 0.298709 \\
\hline H7A2 & 0.473766 & 0.307619 \\
\hline $\mathrm{C} 8 \mathrm{~A}$ & $0.5025(6)$ & $0.3649(4)$ \\
\hline $\mathrm{C9A}$ & $0.4868(6)$ & $0.3419(4)$ \\
\hline H9A & 0.405706 & 0.340904 \\
\hline $\mathrm{C} 10 \mathrm{~A}$ & $0.5249(5)$ & $0.2571(4)$ \\
\hline $\mathrm{C} 11 \mathrm{~A}$ & $0.5261(6)$ & $0.4092(4)$ \\
\hline $\mathrm{H} 11 \mathrm{~A}$ & 0.507064 & 0.394986 \\
\hline H11B & 0.606732 & 0.412310 \\
\hline $\mathrm{C} 12 \mathrm{~A}$ & $0.4788(6)$ & $0.4916(4)$ \\
\hline $\mathrm{H} 12 \mathrm{~A}$ & 0.399135 & 0.491731 \\
\hline $\mathrm{C} 13 \mathrm{~A}$ & $0.4928(6)$ & $0.5124(4)$ \\
\hline $\mathrm{H} 13 \mathrm{~A}$ & 0.573201 & 0.513146 \\
\hline C14A & $0.4428(6)$ & $0.4464(4)$ \\
\hline C15A & $0.4576(7)$ & $0.4847(4)$ \\
\hline $\mathrm{H} 15 \mathrm{~A}$ & 0.532676 & 0.476261 \\
\hline H15B & 0.404854 & 0.462515 \\
\hline $\mathrm{C} 16 \mathrm{~A}$ & $0.4352(7)$ & $0.5737(4)$ \\
\hline $\mathrm{H} 16 \mathrm{~A}$ & 0.360857 & 0.587340 \\
\hline H16B & 0.488516 & 0.605706 \\
\hline C17A & $0.4460(6)$ & $0.5915(4)$ \\
\hline $\mathrm{H} 17 \mathrm{~A}$ & 0.370449 & 0.597739 \\
\hline C18A & $0.6287(6)$ & $0.3727(4)$ \\
\hline $\mathrm{H} 18 \mathrm{~A}$ & 0.660189 & 0.320343 \\
\hline $\mathrm{H} 18 \mathrm{~B}$ & 0.667210 & 0.399861 \\
\hline $\mathrm{H} 18 \mathrm{C}$ & 0.636320 & 0.402675 \\
\hline C19A & $0.6537(5)$ & $0.2484(4)$ \\
\hline $\mathrm{H} 19 \mathrm{~A}$ & 0.671790 & 0.201012 \\
\hline $\mathrm{H} 19 \mathrm{~B}$ & 0.684227 & 0.294351 \\
\hline $\mathrm{H} 19 \mathrm{C}$ & 0.684756 & 0.244520 \\
\hline$C 20 A$ & $0.5094(7)$ & $0.6696(5)$ \\
\hline $\mathrm{C} 21 \mathrm{~A}$ & $0.6352(6)$ & $0.6601(4)$ \\
\hline $\mathrm{H} 21 \mathrm{~A}$ & 0.669303 & 0.712012 \\
\hline $\mathrm{H} 21 \mathrm{~B}$ & 0.645550 & 0.632574 \\
\hline $\mathrm{H} 21 \mathrm{C}$ & 0.668965 & 0.629885 \\
\hline
\end{tabular}

Table 2 (continued)

\begin{tabular}{|c|c|c|c|c|}
\hline Atom & $x$ & $y$ & $z$ & $U_{\text {iso }} * / U_{\text {eq }}$ \\
\hline$C 22 \mathrm{~A}$ & $0.4594(7)$ & $0.7389(4)$ & $0.4201(2)$ & $0.067(3)$ \\
\hline $\mathrm{H} 22 \mathrm{~A}$ & 0.378833 & 0.734233 & 0.420584 & $0.080^{\star}$ \\
\hline H22B & 0.483481 & 0.734580 & 0.388412 & $0.080^{*}$ \\
\hline$C 23 A$ & $0.4919(8)$ & $0.8215(4)$ & $0.4383(3)$ & $0.079(3)$ \\
\hline $\mathrm{H} 23 \mathrm{~A}$ & 0.571673 & 0.829324 & 0.435098 & $0.095^{\star}$ \\
\hline H23B & 0.454100 & 0.862722 & 0.420808 & $0.095^{\star}$ \\
\hline C24A & $0.4589(7)$ & $0.8272(5)$ & $0.4888(3)$ & $0.069(3)$ \\
\hline $\mathrm{H} 24 \mathrm{~A}$ & 0.483376 & 0.878406 & 0.500925 & $0.083^{*}$ \\
\hline H24B & 0.378251 & 0.825230 & 0.491278 & $0.083^{\star}$ \\
\hline$C 25 A$ & $0.5086(7)$ & $0.7607(5)$ & $0.5176(3)$ & $0.052(2)$ \\
\hline$C 26 \mathrm{~A}$ & $0.4507(6)$ & $0.7534(5)$ & $0.5636(2)$ & $0.072(3)$ \\
\hline $\mathrm{H} 26 \mathrm{~A}$ & 0.374308 & 0.738335 & 0.558887 & $0.108^{\star}$ \\
\hline $\mathrm{H} 26 \mathrm{~B}$ & 0.453697 & 0.803836 & 0.579293 & $0.108^{*}$ \\
\hline $\mathrm{H} 26 \mathrm{C}$ & 0.487398 & 0.713291 & 0.581744 & $0.108^{\star}$ \\
\hline C27A & $0.6342(6)$ & $0.7761(5)$ & $0.5267(3)$ & $0.077(3)$ \\
\hline $\mathrm{H} 27 \mathrm{~A}$ & 0.666444 & 0.729996 & 0.541212 & $0.116^{\star}$ \\
\hline H27B & 0.642407 & 0.821771 & 0.546385 & $0.116^{*}$ \\
\hline $\mathrm{H} 27 \mathrm{C}$ & 0.671445 & 0.786009 & 0.498083 & $0.116^{\star}$ \\
\hline $\mathrm{C} 28 \mathrm{~A}$ & $0.3910(6)$ & $0.0551(4)$ & $0.4623(2)$ & $0.072(3)$ \\
\hline $\mathrm{H} 28 \mathrm{~A}$ & 0.402963 & -0.000607 & 0.468561 & $0.108^{\star}$ \\
\hline $\mathrm{H} 28 \mathrm{~B}$ & 0.381514 & 0.062764 & 0.429868 & $0.108^{*}$ \\
\hline $\mathrm{H} 28 \mathrm{C}$ & 0.325372 & 0.073038 & 0.478116 & $0.108^{*}$ \\
\hline C29A & $0.5967(6)$ & $0.0683(4)$ & $0.4553(2)$ & $0.067(3)$ \\
\hline $\mathrm{H} 29 \mathrm{~A}$ & 0.661444 & 0.097831 & 0.464668 & $0.100^{\star}$ \\
\hline $\mathrm{H} 29 \mathrm{~B}$ & 0.588370 & 0.071813 & 0.422610 & $0.100 *$ \\
\hline $\mathrm{H} 29 \mathrm{C}$ & 0.605067 & 0.013193 & 0.464067 & $0.100^{*}$ \\
\hline C30A & $0.3143(5)$ & $0.4401(4)$ & $0.4334(3)$ & $0.065(2)$ \\
\hline $\mathrm{H} 30 \mathrm{~A}$ & 0.281374 & 0.492282 & 0.431131 & $0.098^{*}$ \\
\hline $\mathrm{H} 30 \mathrm{~B}$ & 0.300365 & 0.418495 & 0.463439 & $0.098^{*}$ \\
\hline $\mathrm{H} 30 \mathrm{C}$ & 0.282205 & 0.405517 & 0.410660 & $0.098^{\star}$ \\
\hline C31A & $0.5342(7)$ & $0.0204(5)$ & 0.5923(3) & $0.052(2)$ \\
\hline N1B & $0.7183(5)$ & $0.4890(3)$ & $0.68917(19)$ & $0.0481(17)$ \\
\hline N2B & $0.6965(5)$ & $0.5467(3)$ & $0.6161(2)$ & $0.068(2)$ \\
\hline $\mathrm{H} 2 \mathrm{~B} 1$ & 0.712595 & 0.593948 & 0.625804 & $0.081^{\star}$ \\
\hline $\mathrm{H} 2 \mathrm{~B} 2$ & 0.680854 & 0.539180 & 0.587628 & $0.081^{*}$ \\
\hline 01B & $0.6989(5)$ & $-0.0377(3)$ & $0.70210(17)$ & $0.084(2)$ \\
\hline $\mathrm{H} 1 \mathrm{~B}$ & 0.701687 & -0.082627 & 0.713356 & $0.101^{\star}$ \\
\hline O2B & $0.7016(4)$ & $-0.1729(3)$ & $0.74797(16)$ & $0.0499(14)$ \\
\hline S2B & $0.66584(19)$ & $0.38746(13)$ & $0.62595(6)$ & $0.0603(6)$ \\
\hline C1B & $0.6787(7)$ & $0.2658(4)$ & $0.6939(2)$ & $0.052(2)$ \\
\hline H1B1 & 0.707128 & 0.233908 & 0.668653 & 0.062 * \\
\hline H1B2 & 0.601480 & 0.250966 & 0.699036 & $0.062^{\star}$ \\
\hline $\mathrm{C} 2 \mathrm{~B}$ & $0.6858(6)$ & $0.3521(4)$ & $0.6821(2)$ & $0.050(2)$ \\
\hline C3B & $0.7121(6)$ & $0.4140(4)$ & $0.7092(2)$ & $0.044(2)$ \\
\hline C4B & $0.7354(7)$ & $0.4027(4)$ & $0.7600(2)$ & $0.051(2)$ \\
\hline C5B & $0.7173(6)$ & $0.3130(4)$ & $0.7735(2)$ & $0.0403(18)$ \\
\hline H5B & 0.636320 & 0.307983 & 0.777092 & $0.048^{*}$ \\
\hline $\mathrm{C} 6 \mathrm{~B}$ & $0.7640(7)$ & $0.2927(4)$ & $0.8212(2)$ & $0.057(2)$ \\
\hline H6B1 & 0.844795 & 0.292946 & 0.819946 & $0.068^{\star}$ \\
\hline H6B2 & 0.740699 & 0.333371 & 0.842897 & $0.068^{*}$ \\
\hline C7B & $0.7241(7)$ & $0.2112(4)$ & $0.8377(2)$ & $0.056(2)$ \\
\hline H7B1 & 0.643797 & 0.213036 & 0.841666 & $0.068^{\star}$ \\
\hline H7B2 & 0.756924 & 0.200479 & 0.867533 & $0.068^{\star}$ \\
\hline $\mathrm{C} 8 \mathrm{~B}$ & $0.7529(6)$ & $0.1422(4)$ & $0.8053(2)$ & $0.0441(19)$ \\
\hline C9B & $0.7133(6)$ & $0.1654(4)$ & $0.7561(2)$ & $0.0400(18)$ \\
\hline H9B & 0.632037 & 0.169050 & 0.759071 & $0.048^{\star}$ \\
\hline
\end{tabular}


Table 2 (continued)

\begin{tabular}{|c|c|c|c|c|}
\hline Atom & $x$ & $y$ & $z$ & $\boldsymbol{U}_{\text {iso }}{ }^{\star} / \boldsymbol{U}_{\mathrm{eq}}$ \\
\hline C10B & $0.7474(6)$ & $0.2489(4)$ & $0.7375(2)$ & $0.0411(18)$ \\
\hline C11B & $0.7305(6)$ & $0.0967(4)$ & $0.7222(2)$ & $0.052(2)$ \\
\hline $\mathrm{H} 11 \mathrm{C}$ & 0.700563 & 0.112251 & 0.692618 & $0.062^{*}$ \\
\hline H11D & 0.809934 & 0.087760 & 0.718426 & $0.062^{*}$ \\
\hline C12B & $0.6763(7)$ & $0.0188(4)$ & $0.7369(2)$ & $0.053(2)$ \\
\hline H12B & 0.595455 & 0.027254 & 0.738072 & $0.064^{*}$ \\
\hline C13B & $0.7152(6)$ & $-0.0040(4)$ & $0.7843(2)$ & $0.0419(19)$ \\
\hline H13B & 0.796505 & -0.008529 & 0.782229 & $0.050^{\star}$ \\
\hline C14B & $0.6920(6)$ & $0.0635(4)$ & $0.8198(2)$ & $0.0418(19)$ \\
\hline C15B & $0.7285(6)$ & $0.0227(4)$ & $0.8649(2)$ & $0.056(2)$ \\
\hline H15C & 0.809094 & 0.022466 & 0.867735 & $0.067^{\star}$ \\
\hline H15D & 0.696732 & 0.049739 & 0.891280 & $0.067^{\star}$ \\
\hline C16B & $0.6832(6)$ & $-0.0623(4)$ & $0.8606(2)$ & $0.058(2)$ \\
\hline $\mathrm{H} 16 \mathrm{C}$ & 0.610306 & -0.065966 & 0.874884 & $0.070^{*}$ \\
\hline H16D & 0.732851 & -0.099751 & 0.875717 & $0.070^{*}$ \\
\hline C17B & $0.6747(6)$ & $-0.0820(4)$ & $0.8086(2)$ & $0.047(2)$ \\
\hline H17B & 0.595334 & -0.088130 & 0.801393 & $0.057^{\star}$ \\
\hline C18B & $0.8817(5)$ & $0.1297(4)$ & $0.8079(2)$ & $0.058(2)$ \\
\hline H18D & 0.918658 & 0.179937 & 0.803035 & $0.087^{\star}$ \\
\hline $\mathrm{H} 18 \mathrm{E}$ & 0.904370 & 0.092317 & 0.784718 & $0.087^{\star}$ \\
\hline $\mathrm{H} 18 \mathrm{~F}$ & 0.901169 & 0.109196 & 0.837647 & $0.087^{\star}$ \\
\hline C19B & $0.8732(5)$ & $0.2537(4)$ & $0.7233(2)$ & $0.055(2)$ \\
\hline H19D & 0.917699 & 0.265811 & 0.749863 & $0.083^{\star}$ \\
\hline H19E & 0.882828 & 0.295040 & 0.700775 & $0.083^{*}$ \\
\hline $\mathrm{H} 19 \mathrm{~F}$ & 0.896299 & 0.203336 & 0.710688 & $0.083^{*}$ \\
\hline C20B & $0.7320(6)$ & $-0.1609(5)$ & $0.7957(2)$ & $0.045(2)$ \\
\hline C21B & $0.8590(6)$ & $-0.1551(4)$ & $0.8011(3)$ & $0.063(2)$ \\
\hline H21D & 0.890640 & -0.207870 & 0.800951 & $0.094^{\star}$ \\
\hline $\mathrm{H} 21 \mathrm{E}$ & 0.876483 & -0.129102 & 0.829602 & $0.094^{*}$ \\
\hline $\mathrm{H} 21 \mathrm{~F}$ & 0.889394 & -0.124654 & 0.776103 & $0.094^{*}$ \\
\hline C22B & $0.6861(7)$ & $-0.2307(4)$ & $0.8242(2)$ & $0.058(2)$ \\
\hline $\mathrm{H} 22 \mathrm{C}$ & 0.718010 & -0.228400 & 0.854738 & $0.070^{*}$ \\
\hline $\mathrm{H} 22 \mathrm{D}$ & 0.606011 & -0.224680 & 0.827107 & $0.070^{*}$ \\
\hline C23B & $0.7113(7)$ & $-0.3123(4)$ & $0.8032(3)$ & $0.063(2)$ \\
\hline $\mathrm{H} 23 \mathrm{C}$ & 0.677169 & -0.354039 & 0.821638 & $0.076^{*}$ \\
\hline H23D & 0.791239 & -0.321276 & 0.802843 & $0.076^{*}$ \\
\hline C24B & $0.6655(7)$ & $-0.3156(4)$ & $0.7542(3)$ & $0.062(2)$ \\
\hline $\mathrm{H} 24 \mathrm{C}$ & 0.684116 & -0.366826 & 0.740612 & $0.075^{\star}$ \\
\hline H24D & 0.584855 & -0.311183 & 0.755144 & $0.075^{\star}$ \\
\hline C25B & $0.7122(7)$ & $-0.2491(5)$ & $0.7245(3)$ & $0.054(2)$ \\
\hline C26B & $0.6445(7)$ & $-0.2380(5)$ & $0.6806(2)$ & $0.075(3)$ \\
\hline $\mathrm{H} 26 \mathrm{D}$ & 0.568626 & -0.225696 & 0.688362 & $0.112^{\star}$ \\
\hline $\mathrm{H} 26 \mathrm{E}$ & 0.646734 & -0.286462 & 0.662919 & $0.112^{*}$ \\
\hline $\mathrm{H} 26 \mathrm{~F}$ & 0.675646 & -0.194969 & 0.662973 & $0.112^{*}$ \\
\hline C27B & $0.8342(6)$ & $-0.2663(4)$ & $0.7108(3)$ & $0.069(3)$ \\
\hline H27D & 0.865407 & -0.219848 & 0.696317 & $0.104^{\star}$ \\
\hline $\mathrm{H} 27 \mathrm{E}$ & 0.836356 & -0.310633 & 0.689906 & $0.104^{\star}$ \\
\hline $\mathrm{H} 27 \mathrm{~F}$ & 0.876695 & -0.279083 & 0.737796 & $0.104^{*}$ \\
\hline C28B & $0.6484(7)$ & $0.4543(4)$ & $0.7863(2)$ & $0.077(3)$ \\
\hline $\mathrm{H} 28 \mathrm{D}$ & 0.658170 & 0.509625 & 0.778281 & $0.116^{*}$ \\
\hline $\mathrm{H} 28 \mathrm{E}$ & 0.658803 & 0.447693 & 0.818709 & $0.116^{*}$ \\
\hline $\mathrm{H} 28 \mathrm{~F}$ & 0.574508 & 0.437539 & 0.777985 & $0.116^{\star}$ \\
\hline C29B & $0.8510(7)$ & $0.4365(4)$ & $0.7715(2)$ & $0.079(3)$ \\
\hline H29D & 0.907371 & 0.403590 & 0.757612 & $0.119^{\star}$ \\
\hline $\mathrm{H} 29 \mathrm{E}$ & 0.861110 & 0.437049 & 0.804149 & $0.119^{*}$ \\
\hline $\mathrm{H} 29 \mathrm{~F}$ & 0.856963 & 0.490070 & 0.759778 & $0.119 *$ \\
\hline
\end{tabular}

Table 2 (continued)

\begin{tabular}{lrrrr}
\hline Atom & $\boldsymbol{x}$ & $\boldsymbol{y}$ & $\boldsymbol{z}$ & $\boldsymbol{U}_{\text {iso }} / \boldsymbol{U}_{\text {eq }}$ \\
\hline C30B & $0.5629(5)$ & $0.0762(4)$ & $0.8246(2)$ & $0.059(2)$ \\
H30D & 0.525746 & 0.025438 & 0.822721 & $0.089^{*}$ \\
H30E & 0.537093 & 0.110339 & 0.800302 & $0.089 *$ \\
H30F & 0.546889 & 0.100658 & 0.853686 & $0.089 *$ \\
C31B & $0.6960(6)$ & $0.4839(5)$ & $0.6459(3)$ & $0.052(2)$ \\
\hline
\end{tabular}

$(\mathrm{H})=1.5 U_{\text {eq }}(\mathrm{O})$, and with $\mathrm{O}-\mathrm{H}=0.82$ or $0.85 \AA$ ( $(-\mathrm{OH}), U_{\text {iso }}$ $(\mathrm{H})=1.2 U_{\text {eq }}(\mathrm{O})$ (SHELX [1-4]). The methylene and methyl $\mathrm{H}$ atoms were placed in geometrically idealized positions and constrained to ride on their parent $\mathrm{C}$ atoms, with $\mathrm{C}-$ $\mathrm{H}=0.96 \AA(-\mathrm{CH}), 0.97 \AA\left(-\mathrm{CH}_{2}\right), U_{\text {iso }}(\mathrm{H})=1.2 U_{\text {eq }}(\mathrm{C})$ and $\mathrm{C}-\mathrm{H}=0.96 \AA\left(-\mathrm{CH}_{3}\right), U_{\text {iso }}(\mathrm{H})=1.5 U_{\text {eq }}(\mathrm{C})$.

The absolute configuration was verified by a FlackParsons parameter of 0.01(2) based on 1015 quotients [4].

\section{Comment}

20(R)-panaxadiol (PD) is a significant apogenin of ginsenosides, which can be achieved by acid hydrolysis from leaves and stems of ginseng. It has been reported to have strong anticancer activities, and the mechanism involved in inducing apoptosis [5]. It has been reported that 20(R)panaxadiol emerged significant inhibiting effect toward the Du-145, Hela, HepG2, and MCF-7 cells [6]. It is also a potent approach to diversifying the structures of natural products and preparing a variety of derivatives for the search of new leading compounds [7]. PD and panaxatriol type saponins and ophioponins, might exert tremendous cardio-protective effects and anti-inflammatory activities by blocking nuclear factor-kappa B (NF-kB) pathway [8]. Compared with PD, panaxadiol halogen-derivatives showed higher inhibitory activity toward HCT-116, BGC-823,21SW-480 [9]. Consequently, the structural modification of $20(R)$-panaxadiol may be valuable in obtaining new chemical derivatives for pharmacological screening and structure-activity relationship studies.

As shown in figure, there are two molecules in the asymmetric unit of the title structure. Each of them contains one classical intramolecular $\mathrm{O}-\mathrm{H} \cdots \mathrm{O}$ hydrogen bond. Furthermore, the two crystallographically independent molecules are connected by classical intermolecular $\mathrm{N}-\mathrm{H} \cdots \mathrm{O}$ hydrogen bonds. The distances of $\mathrm{H}(1 \mathrm{~B}) \cdots \mathrm{O}(2 \mathrm{~B})$ and $\mathrm{O}(2 \mathrm{~A}) \cdots \mathrm{H}(1 \mathrm{~A})$ are $1.809(34)$ and 1.852(33), respectively. While the distances of $\mathrm{H}(2 \mathrm{~A} 2) \cdots \mathrm{O}(1 \mathrm{~B})$ and $\mathrm{H}(2 \mathrm{~B} 2) \cdots \mathrm{O}(1 \mathrm{~A})$ are 2.261(43) and 2.288(36), respectively. The molecules extends into an infinite two-dimensional network through weak non-classical intermolecular $\mathrm{C}-\mathrm{H} \cdots \mathrm{N}$ hydrogen bond. Bond lengths and angles are in the expected ranges [10, 11]. 
Acknowledgements: X-ray data were collected at School of Chemistry, Chemical Engineering and Environmental Engineering, Weifang University, Weifang, 370700, People's Republic of China. This work was supported by the National Natural Science Foundation of China (no. 81473104) and Shandong graduate education innovation program (SDYY16132).

\section{References}

1. Bruker. APEX2, SAINT and SADABS. Bruker AXS Inc., Madison, WI, USA (2009).

2. Sheldrick, G. M.: SHELXT - integrated space-group and crystal-structure determination. Acta Crystallogr. A71 (2015) 3-8.

3. Sheldrick, G. M.: A short history of SHELX. Acta Crystallogr. A64 (2008) 112-122.

4. Sheldrick, G. M.: Crystal structure refinement with SHELXL. Acta Crystallogr. C71 (2015) 3-8.

5. Lin, X. H.; Cao, M. N.; He, W. N.; Yu, S. W.; Guo, D. A.; Ye, M.: Biotransformation of $20(R)$-panaxadiol by the fungus Rhizopus chinensis. Phytochemistry 105 (2014) 129-134.
6. Chen, G.; Ge, H.; Li, J.; Li, J.; Zhai, X.; Wu, J.; Song, Y.: Microbial transformation of $20(R)$-panaxadiol by Absidia corymbifera AS 3.3387. J. Mol. Catal. B: Enzym. 123 (2016) 154-159.

7. Chen, Y.; Du, Y.; Li, Y.; Wang, X.; Gao, P.; Yang, G.; Fang, Y.; Meng, Y.; Zhao, X.: Panaxadiol saponin and dexamethasone improve renal function in lipopolysaccharide-induced mouse model of acute kidney injury. Plos One 10 (2015) e0134653.

8. Zhu, J.; Liang, Y.; Yue, S.; Fan, G.; Zhang, H.; Zhang, M.: Combination of panaxadiol and panaxatriol type saponins and ophioponins from shenmai formula attenuates lipopolysaccharideinduced inflammatory injury in cardiac microvascular endothelial cells by blocking NF-kappa B pathway. J. Cardiovasc. Pharmacol. 69 (2017) 140-146.

9. Xiao, S.; Chen, S.; Sun, Y.; Zhou, W.; Piao, H.; Zhao, Y.: Synthesis and anti-tumor evaluation of panaxadiol halogen-derivatives. Bioorg. Med. Chem. Lett. 27 (2017) 4204-4211.

10. Xu, Y. R.; Yang, J. J.; Liu, J.; Hou, G. G.; Meng, Q. G.: Synthesis and crystal structures of C24-epimeric 20(R)-ocotillol-type saponins. Acta Crystallogr. C72 (2016) 498-503.

11. Puff, H.; Friedrichs, E.; Habscheid, M.; Quante, G.; Chen, W.-S.: Panaxadiol und panaxatriol-monohydrat. Acta Crystallogr. C 42 (1986) 576-579. 